\title{
Investigation of Fin Spacing for Heat Transfer Enhancement in Cross Flow Over Tubes Between Two Parallel Plates
}

\author{
A. N. Oumer ${ }^{l}$, A. F. Alias ${ }^{1}$ \\ ${ }^{1}$ Faculty of Mechanical Engineering, Universiti Malaysia Pahang, Malaysia \\ Email:nurye@ump.edu.my
}

\begin{abstract}
This research explains the investigation of fin spacing for heat transfer enhancement in the finned tube heat exchanger. The objective of this research to recommend the optimum fin spacing for heat transfer enhancement. Three different types of tube and spacing are identified through the simulation from Ansys software. The data between simulation using Ansys Fluent and published literature were being compared. Graph of total pressure, Nusselt number and total temperature have been plotted to make the comparison. Result obtained showed that were a more significant agreement between the simulation and published literature for both types of the tube which are circular and elliptic. From the analysis, there were considered two types of arrangement for the different types of tube. From that, the aligned arrangement is the best for heat transfer enhancement compared to the staggered. For the effect of spacing, there was three spacing which is $1.7 \mathrm{~mm}, 1.8$ $\mathrm{mm}$, and $2.0 \mathrm{~mm}$ spacing with velocity and the total heat flux is set to be constant $\left(v=1.4 \mathrm{~m} / \mathrm{s} ; q=500 \mathrm{~W} / \mathrm{m}^{2}\right)$. For the circular tube, it can be seen that the wider of the fin spacing gave the best heat transfer enhancement in the heat exchanger. Different from the circular which is $1.8 \mathrm{~mm}$ spacing is the best for heat transfer enhancement. Other types of tube are a flat surface which is comparing with the variations of $N u$ vs $R e$ with different heat flux. Then, the result showed that as the $R e$ is increased the $\mathrm{Nu}$ will also increase. In the other side, it is recommended for future work to do the real model dimension followed to import to the Ansys instead of assuming the model is symmetrical.
\end{abstract}

Keywords Water Turbine, Undershot, Low Flow

Paper type Research paper

\section{INTRODUCTION}

A heat exchanger is an equipment for efficient heat transfer from one medium to another medium. A solid wall may separate both media. It is because to prevent mixing or in the other option, they may be in direct contact. In an air-to-air recuperative heat exchanger, a solid barrier is reacted as a separation from the incoming and outgoing airflows. Suppose that there is a warmer and cooler airflow in temperature between the two airflows, heat from the warmer airflow will be transferred through the barrier to the cooler airflow according to the second law of thermodynamics. This states that heat will move from a hotter medium to a colder medium. It is mostly used in refrigeration, space heating, air conditioning, chemical plants, petrochemical plants, petroleum refineries, power plants, natural gas processing, and sewage treatment. Another example of a heat exchanger is found in an automotive part, which is in an internal combustion engine. An engine coolant flows through radiator coils and air flows the coils, which cools the coolant and heats the incoming air.

According to [1], [2], from their article explaining more about industrial heat exchangers, there are many types of heat exchangers operate in different ways; (i) using different flow arrangements, (ii) different equipment, and (iii) different design features. All heat exchangers have similar functions which are for exchanging heat. Either it is exposed directly or indirectly from a warmer medium to a cooler medium. For increasing the heating and cooling efficiency or improving the flow, this equipment is used such as heat exchanger fans, condensers, belts, coolants, additional tubes and lines, along with other components and equipment work. A finned tube on the original surface of the tube, whether the outer surface or inner surface of processing of some fins, the original surface is extended to form an efficient heat transfer components. The finned tubes, also known as fin tubes or finned tubes, the English name is "Fin Tube" or "Finned Tube", also sometimes called "Extended Surface Tube", which extended surface tube. From an article about 'Fin Tube Heat Exchangers Optimization', by Wen at. all, [3] he stated fins are used in extended surface exchangers.

The considerable difference between liquids' heat transfer coefficients often characterizes the conventional fin-tube exchangers. In a gas-to-liquid exchanger, the heat transfer coefficient on the gas side is commonly lower than that on the liquid side. Fins are used on the gas side to increase the surface area and the heat transfer rate between the heat exchanger surface and the surroundings. The fins are used to decrease the size of heat exchangers. Through the fin cross-section, the 
conduction process took place whereas the convection over the fin surface area takes place in and around the fin. When the fluid that exposed is cooler than the fin (higher temperature), the fin base temperature which is the primary surface is higher than the fin surface temperature. If the convection occurs, the heat transferred to the fin from the ambient fluid, the fin base temperature will be lower than the fin surface temperature. This process is to lower the temperature differences and the heat transfer through the fin. Exchangers with fins are utilized when one fluid stream is at high pressure. Types of material and production technique affected the temperature value. All the above causes that finned tube heat exchangers are used in different thermal systems for applications where heat energy is exchanged between different media. Fin spacing is the distance between fins which are used on fan coils, air coolers, dry coolers, evaporators, and condenser. Increasing or decreasing fin spacing has a major impact on the coil performance. Decreasing the spacing between fins will increase the efficiency of the heat exchanger, and it is an inexpensive solution. Therefore, the optimum solution is to have as little spacing as possible. A fin spacing of 1.5-5.0 mm is used for dry cooling. A fin spacing of 3-7 $\mathrm{mm}$ is used for humid air where there is a risk of condensation. A fin spacing of 6-20 $\mathrm{mm}$ is used in freezer room conditions.

$\mathrm{Li}$ at all., [4] studied that the heat transfer performance and pressure drop for fin-and-tube heat exchanger with longitudinal vortex generators (LVGs) on the fin surface were numerically investigated. Rectangular and delta winglet pairs were punched/mounted on the fin surfaces to enhance the heat transfer of the air-side of the fin-and-tube heat exchangers. The results showed that the Nusselt numbers increased up to $20 \%$ for LVGs on plain fins comparing with plain fins channel without LVGs. The heat transfer enhancement by rectangular winglets was more significant than that of the delta winglets. The rectangular winglet with angle of attack of $25^{\circ}$ showed the best overall performance than any other angles of attack in rectangular winglets configurations.

Hu, Wanling; Su, Mei; Wang, Liangcheng; Zhang, Qiang; Chang, Limin; Liu, Song; Wang, Liangbi [5] [6]have carried out on 'The Optimal Fin Spacing of a Circular Tube Bank Fin Heat Exchangers with Vortex Generators' to find the optimal fin spacing regarding the different front flow velocities of a circular tube bank fin heat exchanger with vortex generators. They said in a real application, fin spacing of tube bank heat exchanger can be adjusted once the pattern of the fin is determined. The velocity of the air flow is not all the same in front of the heat exchangers. Therefore, the effects of the fin spacing are needed on the heat transfer performance of such heat exchangers.

Yeom at. all., [6] have to study heat transfer and pressure drop characteristics of micro pin fin arrays in a narrow rectangular channel with air through flow are studied with different flow rates ranging from laminar to turbulent flow. Copper micro pin fins $150-400 \mu \mathrm{m}$ long and $75-700 \mu \mathrm{m}$ in diameter are fabricated by microfabrication techniques. Performance ratios that compare heat transfer to pressure drop characteristics are evaluated to investigate the performance of the micro pin-fin surfaces when both heat transfer and pressure drop are important. The results indicate that dynamic fluid effects generated around micro pin fins take a more dominant role for heat transfer enhancement than the area increase due to micro pin fins. A maximum heat transfer enhancement of $79 \%$ over a plain surface is achieved due to a micro pin-fin surface with a height of $250 \mu \mathrm{m}$ and a diameter of $400 \mu \mathrm{m}$. It is expected that the micro pin-fin surfaces can be used for improving the cooling performance of fan-assisted heat sinks for electronics thermal management.

Hu at. all., [7] focused on a computational study the performance analysis through a rectangular channel with circular pin fins that attached to a flat surface. Staggered arrangements arranged pin fins and the heat transfer is assumed to be conjugated in nature. The radiation effects and body forces are negligible. Then, the hydrodynamic and thermal behaviors are studied for Reynolds number varying from 200 to 1000 . The increasing of the fin density along the streamwise direction, the heat transfer also increases. The fin materials with large thermal conductivity provide high heat transfer rate with no increase in the pressure drop according to the same surface area and pumping power. In their research, they considered threedimensional rectangular ducts with a staggered array of circular pin fins mounted on the heated bottom wall maintained at a constant temperature which is by computational domain considered. For investigating the streamwise fin spacing, they picked up three different number of spacing $(22,16$, and 13$)$.

Ayli at. all., [8] have investigated the steady-state heat transfer from rectangular fin arrays is examined experimentally and numerically for turbulent fully developed flow. The effects of geometrical parameters on heat transfer coefficient and Nusselt number are investigated. For different inter fin ratios, Reynolds number and Nusselt number dependence of the results is investigated. A generalized empirical correlation for Nusselt number is developed for rectangular fins for a Reynolds number range of $17 \times 107<\operatorname{Re}<2.47 \times 108$, and an aspect ratio of $0.089<\mathrm{d} / \mathrm{w}<0.0625,0.24875<\mathrm{t} / \mathrm{L}<0.729$. The correlation can predict the results with a relative rms error of $11.14 \%$.

The test section is made up of aluminum [9], [10]. The fins are located at the top and the bottom plates through grooves by using silicon rubber to distinguish the air leakage. Electric heaters heat the plates. Four different test section are employed through this research. The development of the length is necessary to achieve a streamwise-periodic flow is a function of the Reynolds number and fin spacing. When reducing the fin spacing is quite similar on decreasing the Reynolds number. Also, the heat transfer coefficient increase with the decreasing the heat flux and increasing the thermal conductivity of the fins. 
A.A. Gholami, Mazlan A.Wahid, H.A. Mohammed [11] have investigated the enhancement heat transfer performance of fin-and-tube compact heat exchangers with different types of vortex generators. This research has been done in two ways; (a) experimental (b) simulation. The heat transfer coefficient of the fin-and-tube compact heat exchanger is improved compared with the baseline case and the conventional form of winglets. The rectangular winglet vortex generators with wavy-up and wavy-down significantly enhance the heat transfer performance of fin-and-tube compact heat exchangers. Also, the results show that using wavy-up rectangular vortex generators (RVG) has the best heat transfer performance and RVG with conventional form has lower heat transfer enhancement in proportion to the wavy RVG.

Sérgio Nascimento Bordalo and Francisco Eduardo Mourão Saboya [12][12] have investigated the determination of pressure drop coefficients (loss coefficients) for elliptic and circular sections in one, two and three-row arrangements of plate fin and tube heat exchangers. The experiments permitted to correlate the dimensionless loss coefficient with the flow Reynolds number in the rectangular channel formed by the plate fins. The experimental technique consisted of the measurement of the longitudinal pressure distribution along the flow channel, for several values of air mass flow rate. The total number of data runs, each one characterized by the flow Reynolds number, was 216 . The present geometry is used in compact heat exchangers for air conditioning systems, heaters, radiators, and others. Also, it is verified the influence of the utilization of elliptic tubes, instead of circular ones, in the pressure drop. The measurements were performed for Reynolds numbers ranging from 200 to 1900 . Within the knowledge of the authors, the results reported in the present research are original. They are applicable to plate fin and tube compact heat exchangers. For Reynolds numbers in the range $1000-1800$, the replacement of the circular tubes by elliptic tubes, with $\mathrm{b} / \mathrm{a}=0.5$, yields a reduction on the loss coefficient of about $30 \%$. A greater reduction on the pressure drop could be obtained by increasing the separation between the tubes.

Tahseen Ahmad Tahseen, M. Ishak, and M. M. Rahman [13] have studied has been made to investigate heat transfer and air flow around a flat tube of in-line flat tube banks with laminar forced convection. Measurements were conducted for sixteen tubes in the flow direction; four tubes in the rows, the three air velocity (0.6, 0.8 and $1.0 \mathrm{~ms}-1)$ and Reynolds number are $\mathrm{ReDh}=527,703$ and 880 , where $\mathrm{Dh}$ is the hydraulic diameter of the tube. The total heat flux supplies in all tubes are 967.92, 2258.48 and $3629.70 \mathrm{~W} \mathrm{~m}-2$, respectively. The study results can be summarized as: (1) As the average Nusselt number of air flow increases with the increase of Reynolds number. (2) The average Nusselt number of all flat tubes is increased 23.7\%-36.7\% with Reynolds numbers varying from 527 to 880 at the fixed heat flux. (3) The pressure drop increase with the increase Reynolds number, also dimensionless pressure drop decreases with the increase Reynolds number. This research explains the investigation of fin spacing for heat transfer enhancement in the finned tube heat exchanger, and objective of this research to recommend the optimum fin spacing for heat transfer enhancement

\section{Circular Tube and Elliptic Tube}

For this tube, we just refer the same journal which is entitled 'Pressure Drop Coefficients for Elliptic and Circular Sections in One, Two and Three-Row Arrangements of Plate Fin and Tube Heat Exchangers' from the Brazilian Society of Mechanical Sciences (1999). The objective of the present work is the experimental determination of pressure drop coefficients (loss coefficients) for elliptic and circular sections in one, two and three-row arrangements of plate fin and tube heat exchangers. For the simulation, we take the model of one-row arrangement for aligned and staggered. To determine the value of the loss coefficient for each run, (fixed airflow Reynolds number) the following definition is employed:

$$
K=\frac{\Delta P d}{\left(N \frac{\rho \mathrm{V}^{2}}{2}\right)}
$$

Where $\Delta \mathrm{Pd}$ is the pressure drop due only to the presence of the tubes (vertical distance between the two parallel straight lines; $\mathrm{N}$ is the number of tube rows; $\rho$ is the air density and $\mathrm{V}$ is the average air frontal velocity. The average air frontal velocity is given the following equation:

$$
V=\frac{R e v}{d}
$$

Where Re is the Reynold number; $v$ is the kinematic viscosity $(v=\mu / \rho ; \mu=$ absolute/dynamic viscosity; $\mu=1.846 \times 10-5 \mathrm{~N}$ $\mathrm{s} / \mathrm{m} 2 ; \rho=$ density; $\rho=1.225 \mathrm{~kg} / \mathrm{m} 3)$; $d$ is the diameter of the circular and elliptic tube section, $\mathrm{m}$.

Although the determination of $\mathrm{K}$, given by Eq. (1), does not require the knowledge of the total pressure $\Delta$ Ptotal, which occurs in the test section, it may be obtained from the following expression:

$\Delta$ Ptotal $=\Delta P d+\Delta P u$ 
where $\Delta \mathrm{Pu}$ is the pressure drop that would occur if the rectangular channel were free from the obstacle caused by the heat exchanger tubes (smooth channel). Such an additional pressure drop is determined from the usual definition of the friction factor, $\mathrm{f}$, expressed as:

$$
f=\Delta P_{u} \frac{2 \delta}{N L} \frac{2}{\rho V^{2}}
$$

where $\delta$ is fin-to-fin distance, $\mathrm{m} ; \mathrm{N}$ is the number of tube rows; $\mathrm{L}$ is the length of the one-row arrangement, $\mathrm{m} ; \rho$ is the air density and $\mathrm{V}$ is the average air frontal velocity.

The friction factor for laminar flow in rectangular channels;

$$
f=\frac{96}{R e}
$$

From the simulation, we took the value of total pressure which the difference of the high and low pressure, and then put the value in the equations that have been given above. The difference between this method are in the journal is $\triangle P d$ value by experimental and we used the simulation which is from Ansys Fluent to get the value of $\Delta$ ptotal.

\section{METHOD}

This investigation needs to be performed in simulations only by using the Ansys software. Firstly, the drawing for the geometry should be getting from the previous research or thesis so that these results of simulation have to be compared with the previous research. Suppose that this investigation has to be done for three different types of tubes. As an example of the tubes shape is circle, ellipse or rectangular. From that, this research gave an idea to compare neither an experimental result or numerical result from other research with the simulation result which is using the Ansys Fluent software. For the first simulation only follow the previous research geometry or dimension and all the data given and comparing both results for the previous thesis and the simulation that has been done. Next, the data from the previous research is taken but only changed the fin spacing.

As an example, if the spacing between the fin to other fin given from the research was $1.7 \mathrm{~mm}$, just changed this fin spacing to $1.8 \mathrm{~mm}$ or $2.0 \mathrm{~mm}$. The spacing taken is taken at least for three spacing. All of this simulation should be done for both arrangements which are for the aligned and staggered arrangement. The result is collected and compared the temperature distribution, Nusselt number, and pressure drop. From all the result that has been collected, we can propose which fin spacing is the best for heat transfer enhancement whether the narrower spacing or wider spacing.

\section{RESULT AND DISCUSSION}

Figure 1 showed the comparison of the published literature with the simulation result of the loss coefficient, $\mathrm{K}$ for the circular tube. From the graph, there was bigger different in its curvature for both results. As we can see, for the journal result showed an increase as the increase of the Reynold number but for the simulation showed the decreased value as the Reynold number is increased. The data from the simulation gave higher value the journal result (refer to Figure 4.2.2.1). To get the value of the loss coefficient, $\mathrm{K}$ there was an equation which is the curve-fitting equations, associated from the tabulation data of the graph in the journal. For the one row arrangement, the equation is $\mathrm{K}=0.0603(\mathrm{Re}) 0.4213$. For the simulation, the result that got is the total pressure which is the differences between the maximum and minimum pressure. Then, the $\Delta \mathrm{Pd}$ got from $\Delta \mathrm{Ptotal}=\Delta \mathrm{Pd}+\Delta \mathrm{Pu}$. 


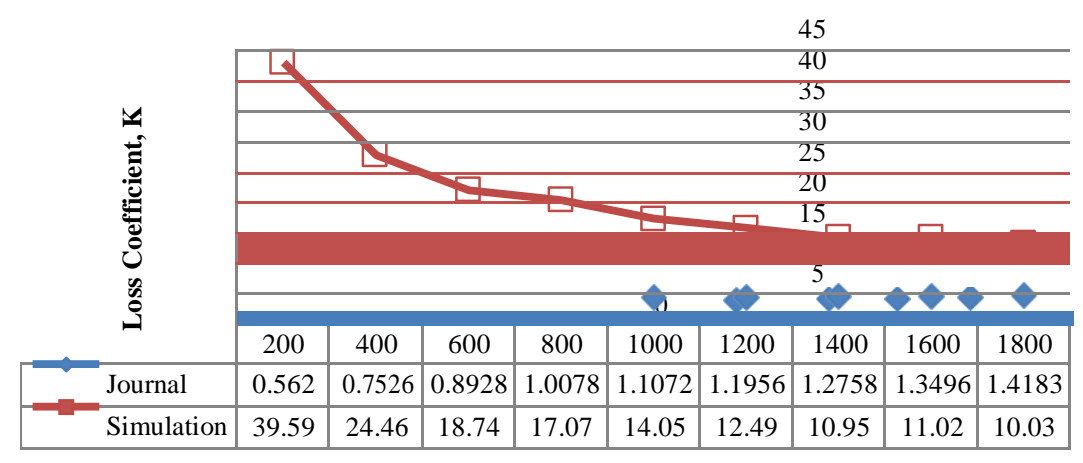

Figure. 1. Comparison of Loss Coefficient, K vs Reynold number (Circular Tube)

Figure 2 showed the comparison of loss coefficient, $\mathrm{K}$ vs Reynold number for the elliptic tube. As we can see, there was a bigger difference between the journal result and the simulation result. As an example at $\operatorname{Re}=200$, the simulation result contributes about 24.08 whereas the journal contributes about 1.9195. However, the differences for both result became closer as we can see at the $\operatorname{Re}=1800$, the simulation gave the value 2.98 and the published journal is about 0.908 . Both results showed the same polar of the data which is as the increased of the Re, the loss coefficient, $\mathrm{K}$ also decreased

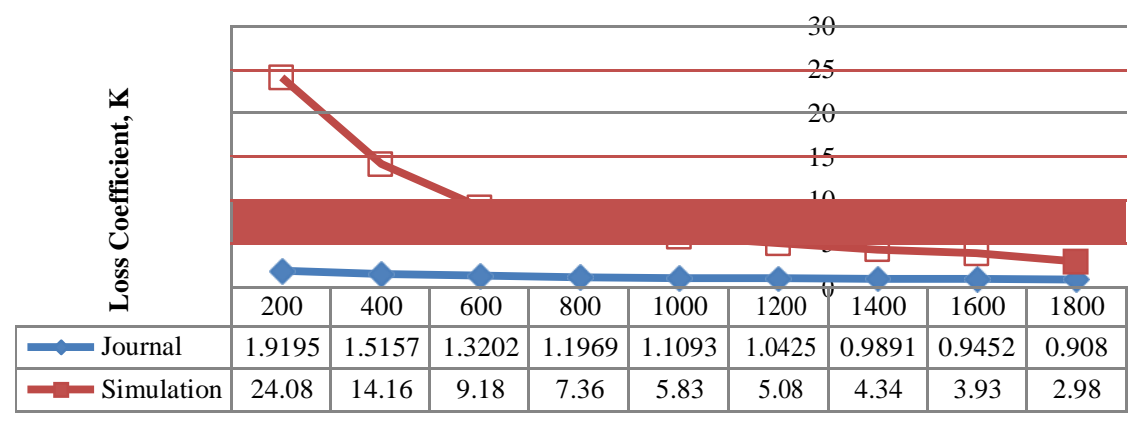

Figure. 2. Comparison of Loss Coefficient, K vs Reynold number (Elliptic Tube)

For this type of tube, we just refer from the published literature entitled "An Experimental Study Air Flow and Heat Transfer over Inline Flat Tube Bank" from Tahseen Ahmad Tahseen, M. Ishak, and M. M. Rahman. This experimental study has been made to investigate heat transfer and air flow around a flat tube of in-line flat tube banks with laminar forced convection. Measurements were conducted for sixteen tubes in the flow direction; four tubes in the rows, the three air velocity $(0.6,0.8$ and $1.0 \mathrm{~m} / \mathrm{s}$ ) and Reynolds number are $\mathrm{ReDh}=527,703$ and 880 , where $\mathrm{Dh}$ is the hydraulic diameter of the tube. The total heat flux supplies in all tubes are $967.92,2258.48$ and $3629.70 \mathrm{~W} / \mathrm{m}^{2}$, respectively.

This research is to investigate the effect for fin spacing to the heat transfer enhancement and the published literature is to investigate heat transfer and air flow around a flat tube of the in-line flat tube. So, the fin spacing is taken about $2.0 \mathrm{~mm}$ spacing and the velocity and the heat flux in all tubes is followed to the journal to see the effect of the heat transfer enhancement.

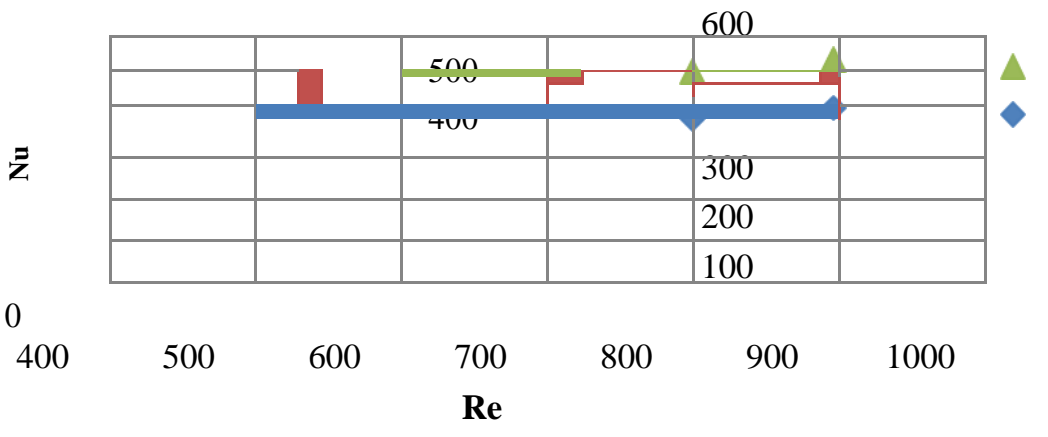

$\mathrm{q}=\wedge 57.92 \mathrm{~W} / \mathrm{m}^{\wedge} 2 \quad \mathrm{q} 2=2258.48 \mathrm{~W} / \mathrm{m}^{\wedge} 2 \quad \mathrm{q} 3=3629.70 \mathrm{~W} / \mathrm{m}^{\wedge} 2$

Figure 3. Variations of $\mathrm{Nu}$ with Re for different heat flux (simulation) 
Figure 3 is the variations of $\mathrm{Nu}$ with Re for different heat flux. As can be seen in the figure above between both graph, there was a bigger difference in value from the published literature and the simulation. From the journal, the polar of the tabulation data with the simulation are the same which are increased in $N u$ as the $R e$ is increased. Actually, from the published journal this project is done by experimental following with some calculations.

\section{EFFECT OF THE FIN SPACING CIRCULAR TUBE}

In the simulation, other than graphs, we can also know the results by looking at the contour of the fin spacing. Contour will show the effect of the overall body of the fin spacing after adding the data according to the journal. In this project, there are many types of contour will be presented, which are, the contour of velocity, the contour of pressure, and contour of density. In this part, we assumed that the velocity and the heat flux are to be the same which are $v=1.4 \mathrm{~m} / \mathrm{s}$ and $q=400 \mathrm{~W} / \mathrm{m}^{2}$. We just assumed the value because to see the effect for the heat transfer enhancement for the fin spacing after it is widening.

The design for fin spacing $1.7 \mathrm{~mm}$ has been tested in simulation the contour also been saved to show the overall operation of the fin spacing. Figures 4 to 5 showed the contour of total pressure for $1.7 \mathrm{~mm}$ spacing of the heat exchanger. Figure 5 showed the center line of the heat exchanger and the maximum pressure captured is 16.57 Pascal and the minimum pressure is 7.25 Pascal. From that, the pressure difference for $1.7 \mathrm{~mm}$ spacing is 9.32 Pascal. As we can see, the total pressure from inlet to outlet is decreased as the air going through the heat exchanger. As we can see above, both figure, Figures 7-8 showed the contour of the total pressure for $1.7 \mathrm{~mm}$ spacing in a staggered arrangement. From the figure, the pressure at the beginning or in the inlet section is the highest value total pressure that got from the simulation. As the air flows going through the heat exchanger for this spacing, its dropped until reaching the outlet of the heat exchanger. Figure 7 showed the exact area which is the higher value still from the inlet section. The value stated from the Ansys is 16.49 Pascal and the value for the exit part which is the outlet is 7.22 Pascal. So, the pressure difference or $\Delta \mathrm{P}$ from both pressure is $9.27 \mathrm{Pascal}$. Then, it is Figure 8 that showed the contour of Nusselt number for $1.7 \mathrm{~mm}$ spacing in an aligned arrangement. From that, the high or maximum value of Nusselt number itself is captured at the first pipe wall which is in red. The value is 1277 that we got from the simulation of the Ansys. Figure 9 is the contour of the Nusselt number for $1.7 \mathrm{~mm}$ spacing in a staggered arrangement. Overall, the maximum or higher value for the Nusselt number is captured at the pipe wall which is the heat flux is supplied there. The exact maximum value is captured actually at the first pipe wall (upper) and second pipe wall (bottom). The value is about 1243 which calculated by the software. Although the temperature is fixed at $300 \mathrm{~K}$, the temperature also has increased after the air-flow reaching the pipe wall as showed in Figure 10 above. However, the temperature before reaching to another pipe wall it slightly dropped in a flat surface and then increased again as the air-flow at the second pipe wall (upper $\&$ bottom). The increment got from the simulation is about $327.29 \mathrm{~K}$ which is this value is the maximum value of total temperature. When the air flows before leaving the pipe wall and going to the flat section became higher in temperature. As we can see in Figure 11 above showed that the contour of total temperature for $1.7 \mathrm{~mm}$ spacing in a staggered arrangement. From that, the total temperature also started to change when the air flows reached the first pipe wall (upper) and continued to the next pipe wall. The highest or maximum total temperature is captured at the last pipe wall which is before the air is leaving the heat exchanger. Also, the temperature is having a slightly dropped before it trying to go to the other pipe wall. So, the maximum total temperature is captured at about 324.18 K. For another fin spacing results are shown in the comparison graph only.

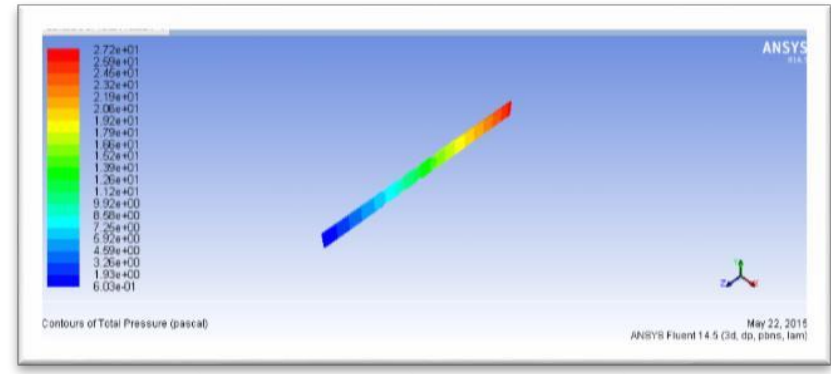

Fig. 4. The contour of Total Pressure for $1.7 \mathrm{~mm}$ fin spacing in an aligned arrangement

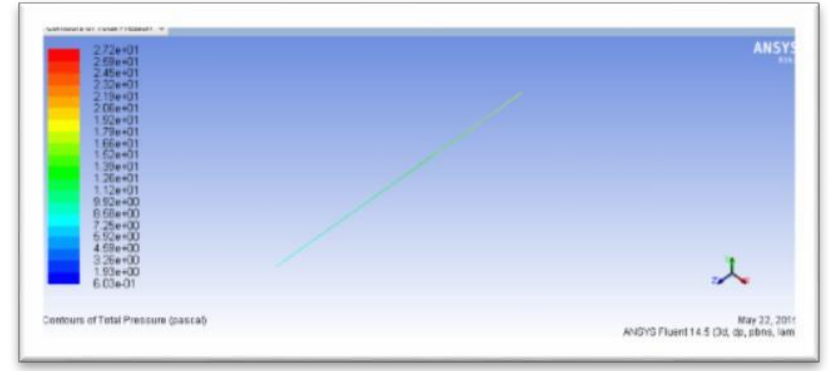

Fig. 5. The contour of Total Pressure for $1.7 \mathrm{~mm}$ spacing in aligned arrangement (centerline) 


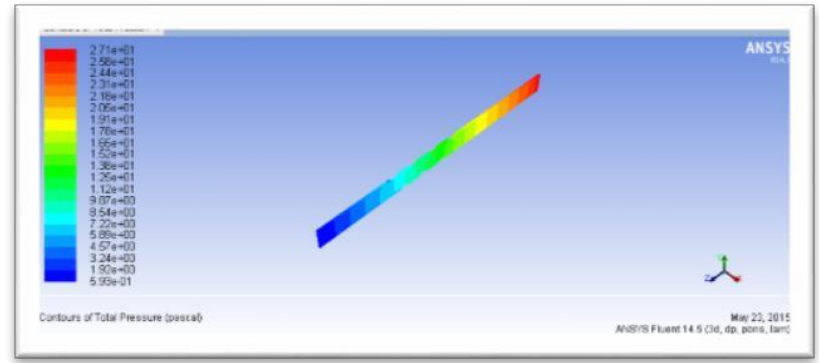

Fig. 6. The contour of total pressure for $1.7 \mathrm{~mm}$ spacing in a staggered arrangement

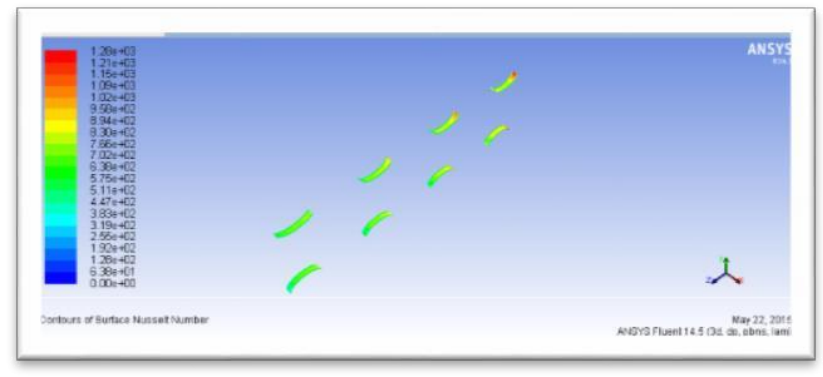

Fig. 8. The contour of Nusselt number for $1.7 \mathrm{~mm}$ spacing in an aligned arrangement

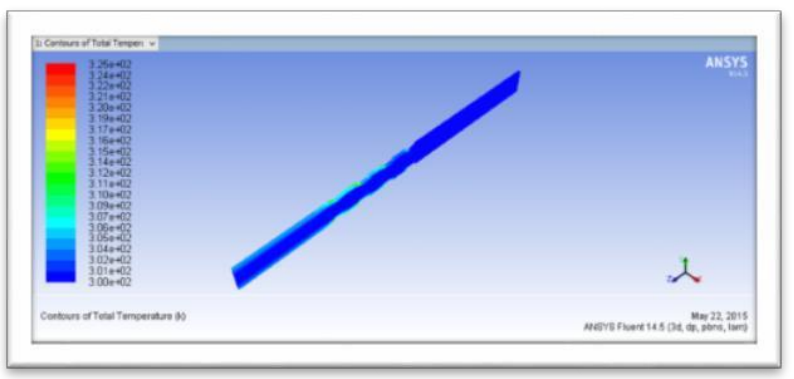

Fig. 10. The contour of total temperature for $1.7 \mathrm{~mm}$ spacing in an aligned arrangement

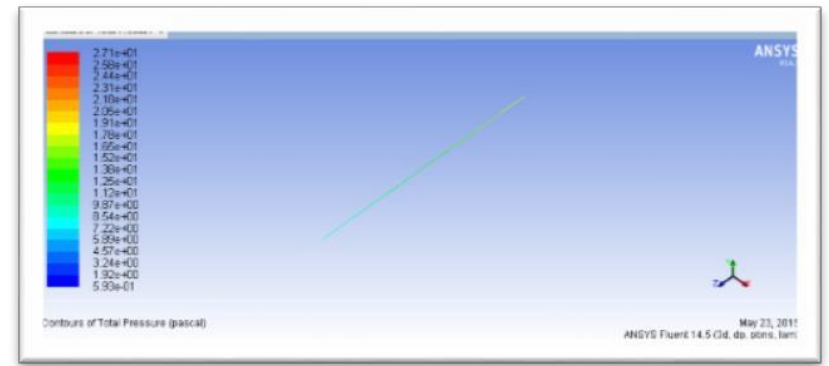

Fig. 7. The contour of total pressure for $1.7 \mathrm{~mm}$ spacing in a staggered arrangement (centerline)

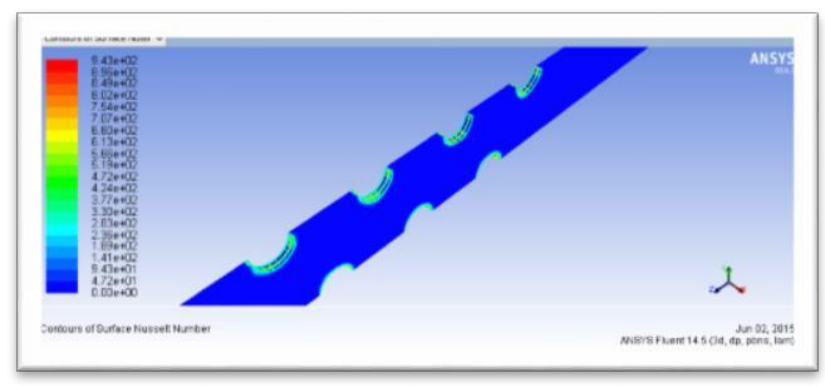

Fig. 9. The contour of Nusselt number for $1.7 \mathrm{~mm}$ spacing in a staggered arrangement

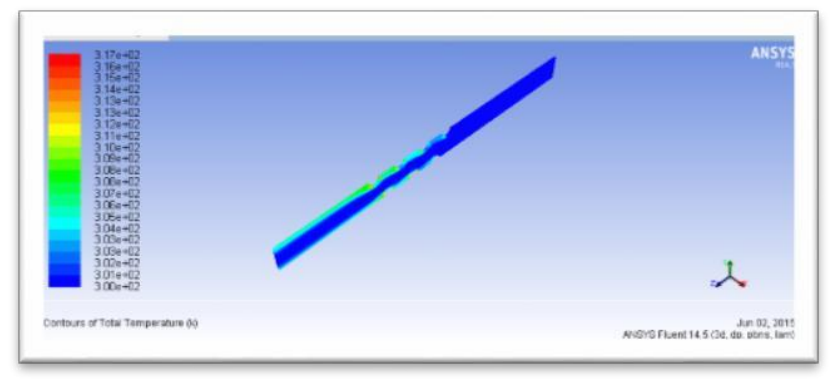

Fig. 11. The contour of total temperature for $1.7 \mathrm{~mm}$ spacing in a staggered arrangement

\section{GRAPH OF COMPARISON THE EFFECT OF SPACING}

Figure 12 showed the comparison of pressure drop for the aligned and staggered arrangement of a circular tube with different fin spacing. From the graph, the value of aligned and staggered arrangement gave a different in its curvature. Although both graphs gave the same polar which is as the fin spacing is increased the pressure drop is also decreased, there is a high value for the tabulation data in the graph for the aligned arrangement compared to the staggered. As we can see here, the aligned arrangement contributes to the higher pressure difference as the air flows going through the heat exchanger. Different from the comparison of Nusselt number (refer Figure 13) which is at the aligned and staggered arrangement curvature. For aligned arrangement, wider of the fin spacing gave the increased in value of the Nusselt number. However, at the staggered highest value of Nusselt number can be seen at the spacing $1.8 \mathrm{~mm}$. The other things, value for the data at the aligned arrangement contributes the highest compared to the staggered. Even though at the beginning, the temperatures of this condition are kept constant which is considered as room temperature $\left(300{ }^{\circ} \mathrm{K}\right)$, the total temperature has changed whether increased or decreased. In this type of tube, both of the arrangement contributed to the same polar which are decreased as the airflow moving from the inlet to the outlet. The differences for both arrangements are the value for the staggered is lower compared to the aligned. The temperature has increased because it absorbed the energy from the pipe walls as there was heat flux supplied at the model of the heat exchanger (refer Figure 14). 


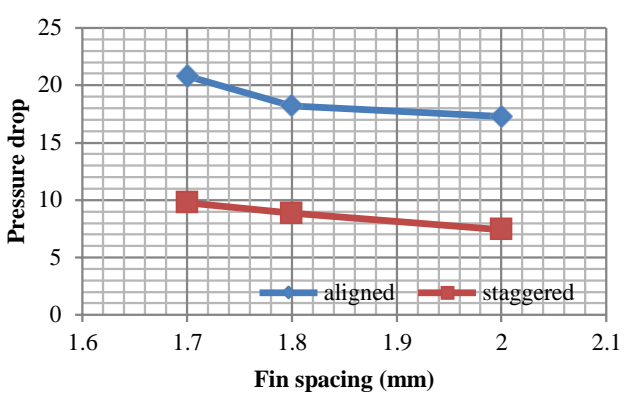

Figure 12. Comparison of Pressure Drop for the aligned and staggered arrangement of a circular tube with different fin spacing

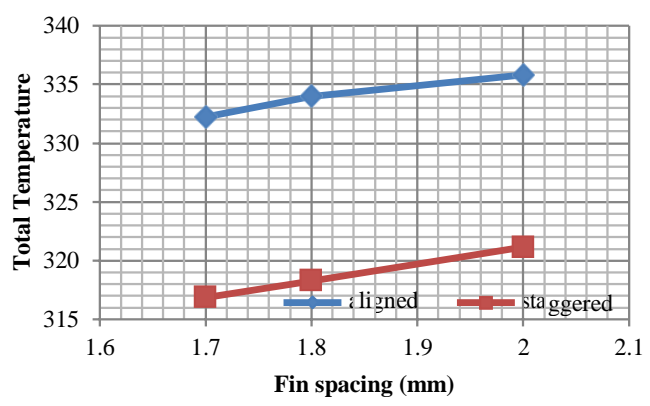

Figure 14. Comparison of total temperature for the aligned and staggered arrangement of a circular tube with different fin spacing

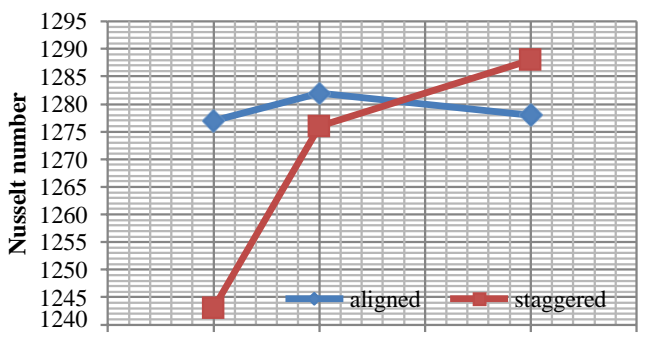

$\begin{array}{llllll}1.6 & 1.7 & 1.8 & 1.9 & 2 & 2.1\end{array}$

Fin spacing $(\mathbf{m m})$

Figure 16. Comparison of Nusselt number for the aligned and staggered arrangement of the elliptic tube with different fin spacing

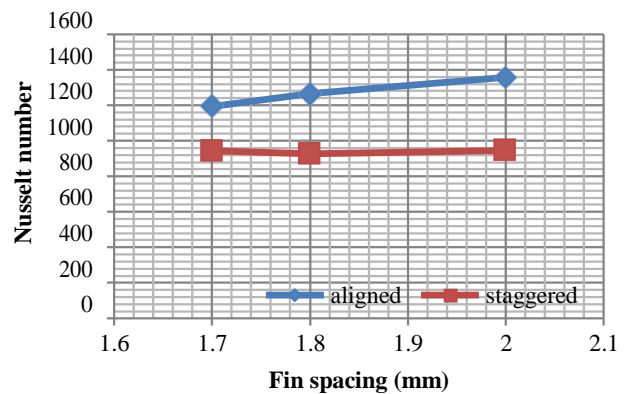

Figure 13. Comparison of Nusselt number for the aligned and staggered arrangement of a circular tube with different fin spacing

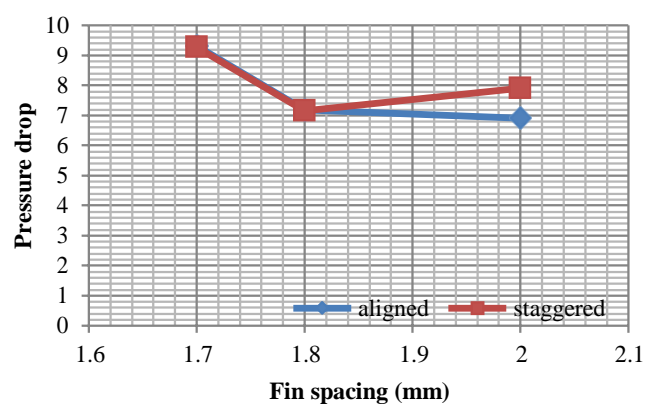

Figure 15. Comparison of pressure drop for the aligned and staggered arrangement of the elliptic tube with different fin spacing
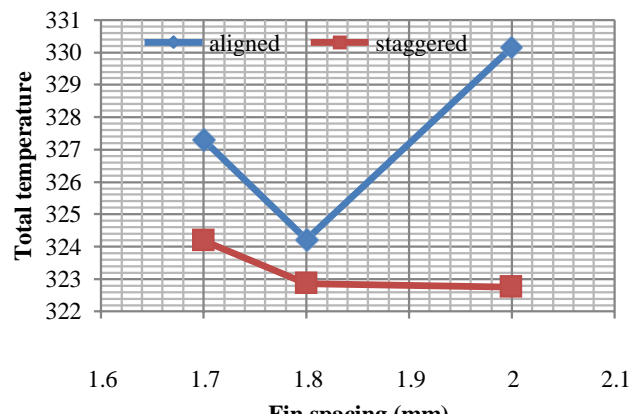

Fin spacing $(\mathbf{m m})$

Figure 17. Comparison of total temperature for the aligned and staggered arrangement of the elliptic tube with different fin spacing

\section{ELLIPTIC TUBE}

Figures 14-15 showed the comparison of total temperature and pressure drop for the aligned and staggered arrangement of the elliptic tube with different fin spacing. It can be seen that the value of aligned and staggered did not give any much different in its curvature. The tabulation data are decreasing in value as the spacing is wider for the aligned arrangement. However, the pressure drop at $1.8 \mathrm{~mm}$ spacing is the lowest compared to $1.7 \mathrm{~mm}$ and $2.0 \mathrm{~mm}$ spacing for the staggered. The value at $2.0 \mathrm{~mm}$ contributes the different as at the aligned is became lower than before and staggered is became higher than the before.

Figure 16 showed a graph of fin spacing vs. Nusselt number with a different arrangement. The staggered arrangement values from the graph are increased as the spacing is wider. Different from the aligned which is the maximum value of Nusselt 
number contributed from the $1.8 \mathrm{~mm}$ spacing with the value 1282 . However, all of the value not so much different in value which means that the increased or decreased is not too much just only different one or two. From the contour of total temperature, the temperature started to change when the air flows at the pipe wall. The temperature increased at the pipe wall and dropped as it in the flat section. From the graph below (refer Figure 17) the temperature is decreased as the spacing of the fin wider except for the $2.0 \mathrm{~mm}$ spacing in an aligned arrangement. At these conditions, the value became increased and it was the maximum value of total temperature compared to the other fin spacing. The changes are too much compared to the other changes which are not too much value.

\section{CONCLUSION}

The simulation was performed with three types of the tube which are circular, elliptic, and flat surface. All of this is compared with other published literature whether the project is done by experimental or simulation. For the circular and elliptic are compared with the same journal which are to determine the loss coefficient, $\mathrm{K}$ and for the flat surface identify the Nusselt number vs. Reynold number with the different heat flux. As we can conclude the objectives, the best or optimum heat transfer enhancement for the circular tube is in an aligned arrangement. As we can see, the pressure drop, Nusselt number, and maximum total temperature are in an aligned arrangement. In the other hand, Nusselt number is increased as the Reynold number is increased when going through the theory. Then, the best fin spacing for the aligned arrangement is the wider spacing which is $2.0 \mathrm{~mm}$ spacing. The wider the spacing gave better the heat transfer enhancement in cross-flow over tubes between two parallel plates. For a flat surface tube, we did not consider the effect of fin spacing. We just standardized the spacing which is $2.0 \mathrm{~mm}$ spacing but only identifies the Nusselt number as the Reynold number is increased with different heat flux. As we can conclude, the higher total heat flux will increase the effect of the Nusselt number. Then, as we referred to the objectives that the best heat transfer enhancement is the higher total heat flux and higher in Reynold number which will contribute to the higher Nusselt number.

\section{REFERENCES}

[1] A. A. Bhuiyan and A. K. M. S. Islam, "Thermal and hydraulic performance of finned-tube heat exchangers under different flow ranges: A review on modeling and experiment," Int. J. Heat Mass Transf., vol. 101, pp. 38-59, 2016.

[2] "Industrial Heat Exchangers: What They Are, How They Work, and Why They Are Needed," SERVICING AMERICA'S ENERGY, 2014. .

[3] J. Wen, H. Yang, X. Tong, K. Li, S. Wang, and Y. Li, "Configuration parameters design and optimization for plate-fin heat exchangers with serrated fin by multi-objective genetic algorithm," Energy Convers. Manag., vol. 117, pp. 482-489, 2016.

[4] L. Li, X. Du, Y. Zhang, L. Yang, and Y. Yang, "Numerical simulation on flow and heat transfer of fin-and-tube heat exchanger with longitudinal vortex generators," Int. J. Therm. Sci., vol. 92, pp. 85-96, 2015.

[5] D.-K. Yang, K.-S. Lee, and S. Song, "Fin spacing optimization of a fin-tube heat exchanger under frosting conditions," Int. J. Heat Mass Transf., vol. 49, no. 15-16, pp. 2619-2625, 2006

[6] T. Yeom, T. Simon, T. Zhang, M. Zhang, M. North, and T. Cui, "Enhanced heat transfer of heat sink channels with micro pin fin roughened walls," Int. J. Heat Mass Transf., vol. 92, pp. 617-627, 2016.

[7] W. Hu et al., "The optimum fin spacing of circular tube bank fin heat exchanger with vortex generators," Heat Mass Transf., vol. 49, no. 9, pp. $1271-1285,2013$.

[8] E. Ayli, O. Bayer, and S. Aradag, "Experimental investigation and CFD analysis of rectangular profile FINS in a square channel for forced convection regimes," Int. J. Therm. Sci., vol. 109, pp. 279-290, 2016.

[9] C.-H. Huang, I.-C. Yuan, and H. Ay, "An experimental study in determining the local heat transfer coefficients for the plate finned-tube heat exchangers," Int. J. Heat Mass Transf., vol. 52, no. 21-22, pp. 4883-4893, 2009.

[10] A. Dewan, P. Patro, I. Khan, and P. Mahanta, "The effect of fin spacing and material on the performance of a heat sink with circular pin fins," Proc. Inst. Mech. Eng. Part A J. Power Energy, vol. 224, no. 1, pp. 35-46, 2010.

[11] A. A. Gholami, M. A. Wahid, and H. A. Mohammed, "Heat transfer enhancement and pressure drop for fin-and-tube compact heat exchangers with wavy rectangular winglet-type vortex generators," Int. Commun. Heat Mass Transf., vol. 54, pp. 132-140, 2014.

[12] S. N. Bordalo and F. E. M. Saboya, "Pressure drop coefficients for elliptic and circular sections in one, two and three-row arrangements of plate fin and tube heat exchangers," J. Brazilian Soc. Mech. Sci., vol. 21, no. 4, pp. 600-610, 1999.

[13] T. A. Tahseen, M. Ishak, and M. M. Rahman, "An Experimental Study Air Flow And Heat Transfer Of Air Over In-Line Flat Tubebank," in International Conference on Mechanical Engineering Research (ICMER2013), 2013, vol. 1, p. 3. 